Recepción: 15/01/2019

Aceptación: 19/02/2019

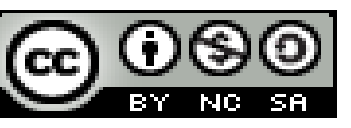

Ciencias de la Salud

Publicación: 05/03/2019

Artículo de investigación

\title{
Disonancias cognitivas en estudiantes universitarios con diabetes tipo II
}

Cognitive dissonances in university students with type II diabetes

Dissonâncias cognitivas em universitários com diabetes tipo II

Mayra Adelina Rivas-Paladines I

adela.alcivar@uleam.edu.ec

Correspondencia: adela.alcivar@uleam.edu.ec

${ }^{\text {I }}$ Doctora en Psicorrehabilitadora y Profesora de Educación Especial, Magister en Salud Mental, Doctora en Psicorrehabilitación y Educación Especial, Docente de la carrera de Psicorrehabilitación y Educación Especial. Universidad Nacional de Loja. Loja. Ecuador. 


\title{
Resumen
}

Se realizó un estudio descriptivo transversal con la finalidad de diagnosticar la situación de los estudiantes de la Universidad de Loja Ecuador que presentan diabetes con problemas de disonancias cognitivas. Para ello se tomó una población de 240 alumnos de dos Áreas de formación académica, la de Educación el Arte y la Comunicación, Jurídica Social y Administrativa, considerando 30 informantes por cada una de las carreras de: Psicorrehabilitación y Educación Especial, Psicología Infantil, Físico Matemáticas y Químico Biológicas, Trabajo Social y Hotelería y Turismo, y 10 profesionales de salud y psicología que laboran en el Departamento de Bienestar universitario; de los cuales se tomó una muestra significativa del $20 \%$ de la población, en un total de 48 estudiantes con problemas de diabetes tipo II. Se concluye que las personas diagnosticadas con diabetes, presentan pensamientos disonantes, sufriendo desequilibrios en su salud mental. El 100\% de los profesionales de salud que trabajan en el Dispensario médico universitario de la UNL, desconocen que los diabéticos presentan disonancias cognitivas para enfrentar la enfermedad y que alteran su salud mental.

Palabras claves: disonancia cognitiva; diabetes juvenil; salud mental; salud integral; calidad de vida.

\begin{abstract}
Cooperative learning is a highly motivating strategy to achieve the learning of another language. Under this notion, this article is presented whose objective is to propose cooperative learning activities for teachers working in the Ecuadorian Public Schools in order to motivate students to learn English. For this, a mixed qualitative-quantitative approach was used. The population was constituted by the students of the eighth year of basic education of the Ecuadorian public schools of the province of Tungurahua and whose sample was of 32 students and 3 English teachers. The applied instrument was a survey of 10 questions, whose alternatives of answers were Yes / No and multiple choice, the analysis of the obtained information allowed to conclude that the English teachers of the Ecuadorian Public Schools investigated did not apply cooperative learning activities in their classes They preferred individual work to facilitate evaluation and make individual comments on the structure of grammar and the use of vocabulary.
\end{abstract}

Keywords: cooperative learning, English language, public education and students. 


\section{Resumo}

A aprendizagem cooperativa é uma estratégia altamente motivadora para alcançar o aprendizado de outra língua. Sob essa noção, este artigo é apresentado com o objetivo de propor atividades de aprendizagem cooperativa para professores que trabalham nas escolas públicas equatorianas, a fim de motivar os alunos a aprender inglês. Para isso, foi utilizada uma abordagem mista qualitativa-quantitativa. A população foi constituída por alunos do oitavo ano do ensino fundamental em escolas públicas província equatoriana de Tungurahua e cuja amostra foi de 32 estudantes e 3 professores de Inglês. O instrumento utilizado foi uma pesquisa com 10 perguntas cujas respostas alternativas eram sim / não e de múltipla escolha, a análise das informações obtidas permitiram concluir que professores de inglês das escolas públicas equatorianas investigadas não se aplicam actividades de aprendizagem cooperativa em suas aulas Eles preferiram o trabalho individual para facilitar a avaliação e fazer comentários individuais sobre a estrutura da gramática e o uso do vocabulário.

Palavras-chave: aprendizagem cooperativa, língua inglesa, educação pública e estudantes.

\section{Introducción}

Es considerada la diabetes mellitus (DM) un síndrome que se caracteriza por una hiperglucemia crónica debida a defectos en la secreción, acción de la insulina o en ambos. (Vicente E, Rodríguez AL, Sánchez E, Quintana L, Riverón JM, Ledo D 2012; p.407-56)

Los pacientes con diabetes mellitus tipo 2 constituyen cerca del 85 a $95 \%$ del total de la población diabética en los países desarrollados; estos porcentajes son más elevados en los llamados en vías de desarrollo. Esta entidad nosológica, es hoy un serio y común problema de salud mundial, el cual, para la mayoría de los países, ha evolucionado en asociación con rápidos cambios sociales y culturales, envejecimiento de las poblaciones, aumento de la urbanización, cambios en la dieta, reducida actividad física y otros estilos de vida y patrones conductuales no saludables.(Orlandi González O, Álvarez Seijas E, Castelo Elías-Calles L, Hernández Rodríguez J, Rodríguez Anzardo BR, González Calero TM, et al 2010)

Sea cual fuere la causa de la epidemia de diabetes mellitus tipo 2 (DM2), la solución a nivel poblacional se encuentra en todo caso en el cambio en el estilo de vida. Todos los estudios epidemiológicos realizados en los últimos años relacionados con la prevalencia de la DM 
demuestran un importante incremento de ella a nivel mundial. (Vicente Sánchez B, Zerquera Trujillo G, Rivas Alpizar E, Muñoz Cocina J, Gutiérrez Cantero Y, Castañedo Álvarez E. 2010) La disonancia cognitiva es una sensación desagradable causada por sostener dos ideas contradictorias al mismo tiempo. Las "ideas" o "cogniciones" en cuestión pueden ser las actitudes y creencias, así como la sensibilización de comportamiento. La teoría de la disonancia cognitiva propone que las personas tienen un impulso de motivación para reducir la disonancia cambiando sus actitudes, creencias y comportamientos, o justificando o racionalizando sus actitudes, creencias y comportamientos. La teoría de la disonancia cognitiva es una de las teorías más influyentes y ampliamente estudiadas en psicología.

La disonancia normalmente se produce cuando una persona percibe una inconsistencia lógica entre sus cogniciones. Esto sucede cuando una idea implica lo contrario de otra. (Disonancia Cognitiva. 2012).

El psicólogo León Festinger propuso la teoría de la disonancia cognitiva que explica cómo las personas intentan mantener su consistencia interna. Sugirió que los individuos tienen una fuerte necesidad interior que les empuja a asegurarse de que sus creencias actitudes y su conducta son coherentes entre sí. Cuando existe inconsistencia entre estas el conflicto conduce a la falta de armonía algo que la gente se esfuerza por evitar. (Disonancia Cognitiva 2014)

Esta teoría ha sido ampliamente estudiada en el campo de la psicología puede definirse como la incomodidad tensión o ansiedad que experimentan los individuos cuando sus creencias o actitudes entran en conflicto con lo que hacen. Este displacer puede llevar a un intento de cambio de la conducta o a defender sus creencias o actitudes e incluso llegando al autoengaño para reducir el malestar que producen. (Disonancia Cognitiva 2014)

Es obvio que una buena Salud Mental frente al diagnóstico de las enfermedades, es clave para gozar de una buena salud integral; sin embargo, somos nosotros los que tenemos en nuestras manos la herramienta, para evitar en gran medida los problemas mentales causado por la falta de concienciación en el cuidado de la salud ya que los diabéticos por la no aceptación de su enfermedad comprometen emocionalmente a la familia para quienes no le es fácil aceptar y aprender a convivir y asumir la responsabilidad de cuidado frente al diabético, sin contar con la colaboración y empoderamiento de la comunidad e instituciones educativas . 
Con la finalidad de detectar y atender los casos con diabetes y disonancia cognitiva en el control de la enfermedad se realizó esta investigación en estudiantes diabéticos en la Universidad Nacional de Loja. Ecuador.

\section{Material y Método}

Se realizó un estudio descriptivo transversal con la finalidad de diagnosticar la situación de los estudiantes de la Universidad de Loja Ecuador que presentan diabetes con problemas de disonancias cognitivas.

Para ello se tomó una población de 240 alumnos de dos Áreas de formación académica, la de Educación el Arte y la Comunicación, Jurídica Social y Administrativa, considerando 30 informantes por cada una de las carreras de: Psicorrehabilitación y Educación Especial, Psicología Infantil, Físico Matemáticas y Químico Biológicas, Trabajo Social y Hotelería y Turismo, y 10 profesionales de salud y psicología que laboran en el Departamento de Bienestar universitario; de los cuales se tomó una muestra significativa del $20 \%$ de la población, en un total de 48 estudiantes con problemas de diabetes tipo II .

Con la observación se logró extraer datos relevantes, mediante la deducción de leyes, principios y conceptos generales. Se utilizaron técnicas e instrumentos como la entrevista, la encuesta, que permitieron la operacionalización de las variables y contrastar con lo que dicen los entrevistados, de su enfermedad y del servicio que reciben del Dispensario médico universitario y, finalmente con el cuestionario de las disonancias cognitivas, se determinó las disonancias que presenta al enfrentar la diabetes tipo II.

Se utilizó como medida de resumen el por ciento.

\section{Resultados}

Al encuestar a los profesionales con respecto al conocimiento que poseen acerca de la situación de los estudiantes universitarios que presentan diabetes y disonancias cognitivas el $100 \%$ de los profesionales aseguran no saber

Los resultados obtenidos a través de la entrevista al total de estudiantes (240) universitarios con diabetes mellitus tipo II indicaron 60 estudiantes que corresponden al 25\% señalan que influyo en su comportamiento, 55 estudiantes que pertenecen al 23\% manifiestan que influyo en sus pensamientos, otros 55 estudiantes que equivalen al 23\% aseguran que influyo en su salud 
mental, 40 estudiantes que corresponden al 17\%, señalan que influyo en sus hábitos de alimentación, 15 de ellos que pertenecen al 6\% expresan que el diagnóstico de la diabetes influyo en su situación económica, 10 estudiantes que corresponden al 4\% señalan que influyo en sus relaciones sociales y 5 de ellos expresan que influyo en su aprendizaje. (Tabla 1)

\section{Tabla 1}

\section{Influencia del diagnóstico de la enfermedad en los estudiantes universitarios.}

\begin{tabular}{lll}
\hline DIAGNOSTICO DE LA ENEFRMEDAD & F & \% \\
\hline Su comportamiento & 60 & $25 \%$ \\
Sus pensamientos & 55 & $23 \%$ \\
Sus hábitos alimenticios & 40 & $17 \%$ \\
En su salud mental & 55 & $23 \%$ \\
En su situación económica & 15 & $6 \%$ \\
En sus relaciones sociales & 10 & $4 \%$ \\
En su aprendizaje & 5 & $2 \%$ \\
\hline TOTAL & 240 & $100 \%$ \\
\hline
\end{tabular}

Fuente: Elaboración propia

$\mathrm{Al}$ analizar la distribución de los estudiantes que intervinieron en el estudio según sexo $(\mathrm{n}=48)$ no fue significativa $47,9 \%$ femenino y $52,0 \%$ masculino.

En relación con los años de evolución de la enfermedad y el nivel de conocimientos, se comprobó que el mayor número de estudiantes estaban entre 5 y 10 años de evolución de la enfermedad, con 45,8 \%, solamente el 8,3\% posee un nivel insuficiente de conocimientos sobre la diabetes mellitus. En sentido general el $66,6 \%$ de los estudiantes tiene un nivel suficiente de conocimientos. (Tabla 2).

\section{Tabla 2}

Distribución de estudiantes según nivel de conocimiento sobre diabetes mellitus tipo II y años de evolución de la enfermedad.

\begin{tabular}{|c|c|c|c|c|c|c|c|c|}
\hline \multirow{3}{*}{$\begin{array}{c}\text { Años de } \\
\text { evolución }\end{array}$} & \multicolumn{4}{|c|}{ Nivel de conocimiento } & \multicolumn{4}{|c|}{ Total } \\
\hline & \multicolumn{2}{|c|}{ Suficiente } & \multicolumn{2}{|c|}{ Necesario } & \multicolumn{2}{|c|}{ Insuficiente } & \multirow[b]{2}{*}{ No } & \multirow[b]{2}{*}{$\%$} \\
\hline & No & $\%$ & No & $\%$ & No & $\%$ & & \\
\hline $\begin{array}{c}\text { Menor } 5 \\
\text { años }\end{array}$ & 13 & 40.6 & 4 & 33.3 & 3 & 75.0 & 20 & 41.6 \\
\hline $\begin{array}{c}5 \text { a } 10 \\
\text { años }\end{array}$ & 15 & 46.8 & 6 & 50.0 & 1 & 25.0 & 22 & 45.8 \\
\hline $\begin{array}{l}\text { Mayor } 10 \\
\text { años }\end{array}$ & 4 & 12.5 & 2 & 16.6 & - & - & 6 & 12.5 \\
\hline Total & 32 & 66.6 & 12 & 25.0 & 4 & 8.3 & 48 & 100 \\
\hline
\end{tabular}

Fuente: Elaboración propia 
Se observa en la tabla 3 que 24 estudiantes diabéticos que corresponden al 50\% presentan un tipo de tendencia a conflictos de aproximación evitación múltiples con una magnitud de disonancia permanente, 8 estudiantes diabéticos, exteriorizan indecisión frente a distintas situaciones de grupo con una magnitud de disonancia leve, 5 estudiantes diabéticos revelan un tipo de tendencia a cumplimiento forzoso con una magnitud de disonancia moderada y tan solo 11 estudiantes con diabetes que equivalen al $23 \%$ muestran un tipo de actitud positiva que genera disonancia reducida con una magnitud de actitud consonante. Los datos revelaron los estudiantes universitarios con diabetes presentan disonancias cognitivas.

\section{Tabla 3}

\section{Disonancias cognitivas en estudiantes con diabetes mellitus tipo II}

\begin{tabular}{|c|c|c|c|}
\hline TIPO & MAGNITUD & $\mathbf{F}$ & $\%$ \\
\hline Actitud positiva que genera disonancia reducida & Actitud Consonante & 11 & $23 \%$ \\
\hline Indecisión frente a distintas situaciones de grupo & Disonancia leve & 8 & $17 \%$ \\
\hline Tendencia a cumplimiento forzoso & Disonancia moderada & 5 & $10 \%$ \\
\hline Tendencia a conflicto de aproximación evitación múltiples & $\begin{array}{l}\text { Disonancia } \\
\text { permanente }\end{array}$ & 24 & $50 \%$ \\
\hline TOTAL & & 48 & $100 \%$ \\
\hline
\end{tabular}

De los 48 estudiantes estudiados el 24,0\% expresa una atención no adecuada por parte del Dispensario Médico Universitario.

\section{Discusión}

Los resultados obtenidos de la investigación, permite realizar un contraste de criterios y comparaciones científicas, para determinar las disonancias cognitivas en los estudiantes diagnosticados con diabetes.

Según Collera Rives S y colaborados (2016) en su investigación mostró alteraciones en las funciones cognitivas en los pacientes diabéticos tipo 2. Así mismo estudios León Festinger,(1982) realizó sus propios experimentos en diferentes grupos de gente, acerca de las disonancias cognitivas, y comparando los estudios realizados por éstos estudiosos de la psicología, un buen porcentaje de estudiantes universitarios acerca de la influencia psicológica que tuvo en ellos el diagnóstico de la diabetes, señalaron que influyó en sus pensamientos y en su comportamiento, que les causó tendencia a conflictos de aproximación y evitación múltiples, a la 
tendencia de cumplimientos forzosos del control de la enfermedad e indecisión frente a distintos tipo de tentación alimenticia a la que estaban acostumbrados.

La diabetes es considerada como una "condición de vida", antes que una enfermedad. Se evidencia que las personas con diabetes requieren tener un estilo de vida específico, hábitos alimenticios apropiados y los cuidados necesarios en todo momento para así poder mantenerse saludables. Esto requiere que las personas con diabetes sepan muy bien cómo manejar todos estos aspectos de la manera más efectiva, para lograr los mejores resultados en su control. Para que esto sea posible, las personas enfermas deben recibir una adecuada educación diabetológica. Una persona con diabetes que adquiere los conocimientos y educación diabetológica necesarios sabe qué hacer para evitar la aparición de complicaciones tanto agudas como crónicas y cómo atacar a tiempo cualquier problema que se pueda presentar, de manera que logra disfrutar de una vida saludable, productiva, feliz y libre de complicaciones. Existen diferentes formas de adquirir la educación que toda persona con diabetes necesita. Este proceso educativo debe ser continuo e integral. Lo ideal es que utilice todos los medios posibles para mantenerse cada día más educado (a) e informado (a), como complemento de lo que el médico y el equipo multidisciplinario de salud (educador, nutricionista, podólogo, etc.) debe enseñar en materia de diabetes. (Barón P, Márquez E 2010), (Vicente Sánchez B, Zerquera Trujillo G, Rivas Alpizar E, Muñoz Cocina J, Gutiérrez Cantero Y, Castañedo Álvarez E. 2010). El estudio reveló más del 50,0 \% de suficiente conocimiento acerca de la patología en estudio.

Herrera Vásquez y Gómez Urrutia (2015) afirman que la educación debe crear las condiciones para que el estudiante sea educado en el empoderamiento de su propio ser: conectado con su identidad, adquiriendo conocimientos con sentido, creativo e íntegro, de tal modo que pueda decidir su proyecto de vida asociado a su realización personal, clave, finalmente, en la sensación de bienestar subjetivo y de felicidad.

Aun cuando existe un reconocimiento de la importancia de la salud mental en el campo de la salud pública y de la amplia utilización de este concepto en los ámbitos profesionales, académicos y políticos, no obstante enfrenta importantes dificultades epistemológicas y prácticas para precisar qué se entiende por lo "mental", cómo se relacionan "la salud" y "lo mental" y, en consecuencia, cómo se investiga e interviene en el ámbito de la salud mental, puesto que, en la

Pol. Con. (Edición núm. 31) Vol. 4, No 3, marzo 2019, pp. 35-44, ISSN: 2550 - 682X 
práctica, la orientación de las acciones y de las investigaciones no obedecen a definiciones "universales", sino a concepciones construidas a partir de los diferentes enfoques de saludenfermedad. (Restrepo O D A., Jaramillo E J C. 2012 p.p 202-211).

La estrategia científica de la propuesta de educación para la salud mental que se propone es susceptible de incidir en una mejor y más funcional integración e inclusión de estas personas de la comunidad universitaria, académica y administrativa para enfrentar retos clínicos y psicológicos de las enfermedades crónicas degenerativas.

\section{Propuesta de estrategia}

- Objetivo: Diseñar un programa educativo para la salud mental en el tratamiento de la disonancia cognitiva en los estudiantes con diabetes tipo II

- Actividades: capacitar al personal médico del dispensario, docentes y estudiantes en el manejo de prevención de enfermedades crónicas degenerativas, salud mental y disonancia cognitiva

- Formas de organización: conferencias, debates, discusiones grupales cursos, talleres con la temática en educación en salud mental, diabetes, estrés y diabetes psicología rente a los diabéticos y otras.

- Evaluación: Frecuente y final

- Resultados esperados: elevado el conocimiento de personal médico del dispensario, estudiante y profesores acerca de la temática, para brindar el apoyo necesario y la debida orientación psicoterapéutica para alcanzar significativos beneficios a nivel personal, familiar y social que propicie un mejoramiento en su modo de actuación.

A partir del proceso de investigación realizado, se evidenció un alto porcentaje de estudiantes diabéticos con una disonancia cognitiva.

\section{Referencias Bibliográficas}

BARON P, MÁRQUEZ E. (2010). Diabetes mellitus tipo 2 en niños y adolescentes. Medicina Interna de México; 26(1): [aprox. 11p]. Recuperado de http://content.ebscohost.com/pdf23_24/pdf/2010/7KO/01Jan10/51924622.pdf?T=P\&P= AN\&K $=51924622 \& S=R \& D=1$ th\&EbscoContent $=$ dGJyMMvl7ESeqLA4zOX0OLCmr0i ep65Ssqu4SrCWxWXS\&ContentCustomer=dGJyMPGnr06vr7JJuePfgeyx44Dt6fIA. 
COLLERA RIVES SHEILA Y, JIMÉNEZ MORALES R M, MACÍAS DELGADO Y, ECHEMENDÍA RODRÍGUEZ M, IZQUIERDO PAZ J. (2016). Alteraciones cognitivas y actividades instrumentales de la vida diaria en pacientes con diabetes mellitus tipo 2 . Gac Méd Espirit, 18(1): 44-54. Recuperado de: http://scielo.sld.cu/scielo.php?script=sci_arttext\&pid=S1608$89212016000100006 \& \operatorname{lng}=\mathrm{es}$.
Disonancia
Cognitiva.
(2012)
Recuperado
de

https://www.bibliotecapleyades.net/ciencia/ciencia_consciousuniverse321.htm

Disonancia Cognitiva (2014). Recuperado de https://www.academia.edu/32693394/Disonancia_cognitiva

HERRERA VÁSQUEZ, LUIS, \& GÓMEZ URRUTIA, VERÓNICA. (2015). DISONANCIAS ENTRE LA EDUCACIÓN ACTUAL YEL DESARROLLO HUMANO: UNA DISCUSIÓN CLAVE. Páginas de Educación, 8(2), 144-157. Recuperado de http://www.scielo.edu.uy/scielo.php?script=sci_arttext\&pid=S1688-

74682015000200006\&lng=es\&tlng=es.

LEÓN. Festinger, 1982. TEORÍA DE LA DISONANCIA COGNOSCITIVA, La Comunicación Humana, en Schramm, Wilbur, Grijalbo, México, Pág. 67

ORLANDI GONZÁLEZ O, ÁLVAREZ SEIJAS E, CASTELO ELÍAS-CALLES L, HERNÁNDEZ RODRÍGUEZ J, RODRÍGUEZ ANZARDO BR, GONZÁLEZ CALERO TM, et al. (2010). Guías cubanas de práctica clínica basadas en la evidencia sobre el pesquisaje, diagnóstico y tratamiento de la diabetes mellitus tipo 2. Ciudad Habana: INEN Recuperado de http://www.sld.cu/galerias/pdf/sitios/pdvedado/gpc_dm2_final.pdf

RESTREPO O DIEGO A., JARAMILLO E JUAN C. (2012). Concepciones de salud mental en el campo de la salud pública. Rev. Fac. Nac. Salud Pública; 30 (2): 202-211. Recuperado de http://www.scielo.org.co/scielo.php?script=sci_arttext\&pid=S0120386X2012000200009\&lng=en.

VICENTE SÁNCHEZ B, ZERQUERA TRUJILLO G, RIVAS ALPIZAR E, MUÑOZ COCINA J, GUTIÉRREZ CANTERO Y, CASTAÑEDO ÁlVAREZ E. (2010). Nivel de conocimientos sobre diabetes mellitus en pacientes con diabetes tipo 2. Medisur, 8(6): 
412-418 Recuperado de http://scielo.sld.cu/scielo.php?script=sci_arttext\&pid=S1727897X2010000600004\&lng=es.

VICENTE E, RODRÍGUEZ AL, SÁNCHEZ E, QUINTANA L, RIVERÓN JM, LEDO D. (2012). Diabetes mellitus. En: Diagnóstico y tratamiento en Medicina Interna. La Habana: Editorial Ciencias médicas; p.407-56. Recuperado de http://www.bvs.sld.cu/libros_texto/diagnostico_ttmo_medintern/diag_ttmo_medinterna_complet o01.pdf 